HADES, A Code for Simulating a Variety of Radiographic Techniques

M. B. Aufderheide, G. Henderson, A. E. Schach von Wittenau, D. M. Slone, A. Barty, H. E. Martz, Jr.

November 1, 2004

Institute of Electrical and Electronics Engineer's 2004 Medical Imaging Conference

Rome, Italy

October 19, 2004 through October 22, 2004 
This document was prepared as an account of work sponsored by an agency of the United States Government. Neither the United States Government nor the University of California nor any of their employees, makes any warranty, express or implied, or assumes any legal liability or responsibility for the accuracy, completeness, or usefulness of any information, apparatus, product, or process disclosed, or represents that its use would not infringe privately owned rights. Reference herein to any specific commercial product, process, or service by trade name, trademark, manufacturer, or otherwise, does not necessarily constitute or imply its endorsement, recommendation, or favoring by the United States Government or the University of California. The views and opinions of authors expressed herein do not necessarily state or reflect those of the United States Government or the University of California, and shall not be used for advertising or product endorsement purposes. 


\title{
HADES, A Code for Simulating a Variety of Radiographic Techniques
}

\author{
Maurice B. Aufderheide, Member IEEE, Gary Henderson, Alexis E. Schach von Wittenau, Dale M. \\ Slone, Anton Barty, and Harry E. Martz, Jr.
}

\begin{abstract}
It is often useful to simulate radiographic images in order to optimize imaging trade-offs and to test tomographic techniques. HADES is a code that simulates radiography using ray tracing techniques. Although originally developed to simulate X-Ray transmission radiography, HADES has grown to simulate neutron radiography over a wide range of energy, proton radiography in the $1 \mathrm{MeV}$ to $100 \mathrm{GeV}$ range, and recently phase contrast radiography using $X$-Rays in the keV energy range. HADES can simulate parallel-ray or cone-beam radiography through a variety of mesh types, as well as through collections of geometric objects. HADES was originally developed for nondestructive evaluation (NDE) applications, but could be a useful tool for simulation of portal imaging, proton therapy imaging, and synchrotron studies of tissue. In this paper we describe HADES' current capabilities and discuss plans for a major revision of the code.
\end{abstract}

\section{INTRODUCTION}

Radiographic image simulation is a useful tool in the field of nondestructive evaluation for a number of reasons. First, it can help the user to anticipate or understand what is seen in radiographic experiments. Second, it can be used to generate test data for use by tomographic reconstruction codes. Third, it can allow the user to optimize the use of radiography for examining particular defects. Until recently, these advantages have not been as obvious in medical imaging because the objects under analysis are not characterized by CAD/CAM models and because many medical imaging techniques do not collect large field of view images in performing reconstructions. Recent developments, such as the use of digital detectors in wide area radiographic imaging[1], and the use of large imaging arrays for spiral CT[2], may make a radiographic simulation tool as advantageous for the medical imaging field as it is in nondestructive evaluation.

The user has two main options in performing radiographic simulation: Monte Carlo techniques, and ray-tracing techniques. Monte Carlo techniques allow the user to study the "full" physics of radiography using many simulation particles to interact with the object, including source generation and detector properties. For X-Rays some Monte Carlo codes with such capability are MCNP[3], COG[4], TART[5], the integrated Tiger series[6], EGS4[7]/BEAM[8], and GEANT[9]. Unfortunately, these codes are relatively slow and cumbersome in the simulation of radiographic images. For a 300x300 image with 1\% Monte Carlo statistical fluctuations, at least $10^{9}$ particles must be transported through the system. While this is becoming feasible with modern massively parallel machines, such capability is not available for routine studies of radiography.

Ray-tracing techniques are very useful for studies requiring a fast turnaround time. In this approach, a bundle of rays connecting the radiographic source and detector is traced through the specified system. Because of the greater simplicity of this technique, ray-tracing simulations are much faster than their Monte Carlo cousins and can be performed on workstations or fast PCs or Macs. XRSIM[10], SINDBAD[11] and HADES are examples of ray tracing radiographic simulation codes.

In this paper we will discuss the HADES radiographic simulation code and apply it to the FORBILD head phantom[12], using three very different radiographic probes. We start with a discussion of the main features of HADES, then describe how we have modeled the FORBILD head phantom. We then describe how HADES models X- and gamma ray radiography, and show simulated radiographs of the head phantom generated with HADES. We describe how HADES models proton radiography and show simulated radiographs of the head phantom. We then describe how HADES models phase effects in X-radiography and show simulated radiographs for a scaled down "head" phantom. We conclude with a discussion of future directions for HADES.

\section{THE HADES CODE}

HADES is a ray-tracing code that we have been developing for the simulation of radiography used in industrial NDE settings. The name HADES was derived from Greek mythology. For the ancient Greeks, "Aı $\delta \eta \varsigma$ " was the underworld, the abode of the dead. In old-fashioned English translations of the Greek classics, the dead in .'Aı $\delta$ ऽ were referred to as shades, or shadows. Since radiography is the science of making and interpreting shadows, HADES was considered an appropriate name for a radiographic simulation code.

HADES can radiograph a variety of meshes and also has a library of solid-body objects such as plates, spheres, cones, cylinders and other complex shapes that can be included in a simulation. Solid geometry operations such as intersections, 
unions and differences allow more complex objects to be built and radiographed. HADES is often run without any mesh, only using combinations of solid body objects, as is the case in all simulations in this paper.

Because we use a wide variety of radiographic probes at Livermore, we have given HADES the capability to support these probes. HADES can simulate absorption radiography for photon energies ranging from roughly $\sim 1 \mathrm{keV}$ to $100 \mathrm{MeV}$. Spectral and monochromatic sources can be simulated by the code. HADES uses the Livermore Evaluated Photon Data Library[13] for X-Ray absorption cross-sections. An example is given in Section IV. HADES can also simulate neutron radiography for neutron energies ranging from roughly thermal energies up to $30 \mathrm{MeV}$. HADES uses the Livermore Evaluated Neutron Data Library[14] for these simulations. Again, the user can specify spectral or monochromatic neutron sources. HADES can also simulate high-energy proton radiography for proton energies ranging from roughly $1 \mathrm{MeV}$ to $100 \mathrm{GeV}$, taking into account Gaussian multiple Coulomb scattering. For this work, HADES uses the Letaw nuclear attenuation cross-sections [15] and Dahl's expression for radiation length[16]. We show an example of this kind of simulation in Section V. One of the most recent capabilities which we have added to HADES is to model phase effects in $\mathrm{X}$-Ray microscopy for energies of roughly $100 \mathrm{eV}$ to $30 \mathrm{keV}$, using data from Lawrence Berkeley's Center for X-Ray Optics[17]. An example of this capability is shown in Section VI. With the importation of higher energy cross sections from the US National Institute of Standards and Technology[18], we will be able to model this type of probe up to $300 \mathrm{keV}$.

One of our goals in the development of HADES has been to accurately simulate the radiographic process, from source to image formation and detection. Thus the code simulates source blur either approximately by projecting this blur onto the detector plane, or by mapping out the finite spot by brute force. Detector blur and spectral response can be included in the simulation several different ways. The user can include a scattering profile in the simulation, but HADES does not independently compute scattering profiles. This computation must be either estimated analytically or computed with a Monte-Carlo code.

Some parts of the code are parallelized, in order to simulate finite spot size using a discreet set of points and to speed up computationally intense simulations, such as ray tracing $3 \mathrm{D}$ meshes. HADES is written in ANSI C and currently runs on Linux and Unix workstations, Macs, and a variety of supercomputers. A port to Windows is currently being investigated.

HADES is strictly a ray tracing code. All of its initial calculations are done in terms of path length. The path length $p$ along a ray is given by $p=\int_{r a y} \rho \mu d l$, where $\rho$ is material density, $\mu$ is any kind of mass absorption coefficient and the integral is along the ray. For a monochromatic X-ray beam without scatter, the transmitted intensity $I$ along the ray is given by $I=I_{0} e^{-p}$, where $\mathrm{I}_{0}$ is the incident intensity along the ray. The main advance in HADES is that many kinds of radiography can be approximated by functions of path length. Post processing steps are used to convert from path lengths to transmitted intensity.

\section{THE FORBILD HEAD PHANTOM}

FORBILD, the Bavarian Center of Excellence for Medical Imaging and Image Processing, is a consortium of 12 industrial and research labs in Germany devoted to advancing research and development in medical imaging. One of the subgroups developed a set of medical phantoms of anthropomorphic body sections with simplified features, as tests for CT systems.

One of the phantoms is a head phantom[12], developed by Günter Lauritsch and Herbert Bruder, which has a variety of features that are suggestive of the human head. Among the features modeled are the cranium, sinus cavity, bones around the sinus cavity, eyes, the ventricle, subdural hematoma, inner occipital protuberance, eyes, and patches with small differences in "density". These objects are all modeled with spheres, ellipsoids, and elliptical cylinders. We have constructed a HADES model, using solid-body objects, from the description given on the FORBILD website. In our HADES model, we have not included the various resolution features in the ear regions of the phantom. These features are excellent tests of CT algorithms and could have been modeled with HADES, but are extremely detailed and are not necessary for the illustration of HADES' capability.

The FORBILD phantoms provide a density for each feature and a $70 \mathrm{keV}$ absorption description in terms of CT numbers (Hounsfield units). Because HADES will be modeling transmission through this object for several different probes and energies, we need the actual elemental composition of each feature, which is not specified in these phantoms. For these studies, we have used ICRU[19] compact bone composition for all bone objects. For all other tissues, we have used ICRU[19] striated muscle composition.

In Figure 1 we show HADES-generated shadows of the head phantom using the major axes as radiographic axes. Here we have set the "brain" tissue density to 0 , so that the other low contrast features will be visible. We have also reproduced the central sagittal, coronal and axial slices from the FORBILD website. The absence of the ear features is obvious. As we illustrate HADES capabilities in later sections, we will use these three views. 


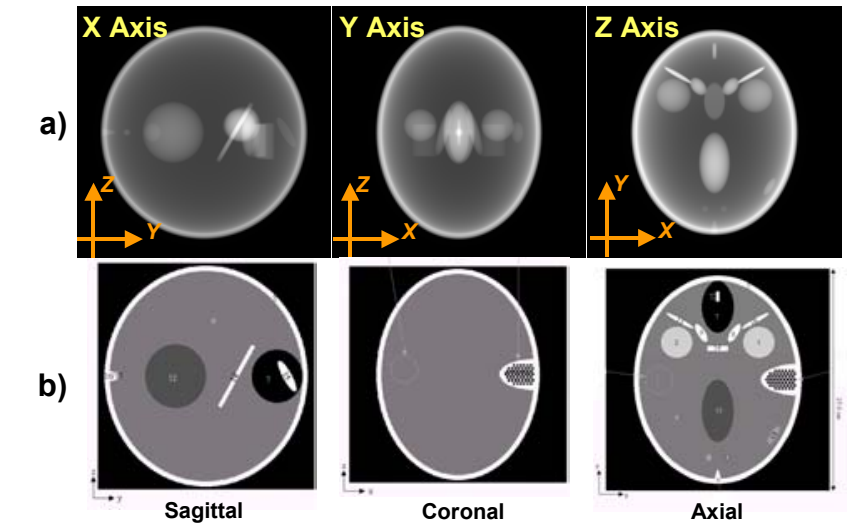

Fig. 1. a) Shadows of the FORBILD head phantom along three axes. b) Central slices through the phantom along each axis.

\section{GAMMA RADIOGRAPHY SIMULATION}

X- and gamma ray sources typically have a broad distribution in energy, often a bremsstrahlung spectrum with $\mathrm{K}$ lines characteristic of the conversion target. Also, detectors have resolution and detection efficiencies that vary with photon energy. In order to accurately model this physics, HADES allows the user to model the relevant energy range with a set of energy bins, specified by the user's definition of the source spectrum. During the simulation, HADES computes a path length image using attenuation coefficients for the center of each energy bin. These images are held within memory.

In order to make full use of HADES' spectral capability, the user must supply at run time two detector files, which specify the detector radial blur as a function of energy and the detector quantum efficiency (DQE) as a function of energy. This information is best calculated by computing the energy deposition in the detector using a Monte Carlo code. Given these files, HADES then blurs each energy bin image appropriately and then combines them using the DQE information. The final output is a single image of the effective path length, properly weighted and blurred by FFT convolution for the spectrum and detector.

If the user specifies a dose from the source, HADES uses a previously computed (with Monte Carlo) dose conversion curve to compute the number of photons in every energy bin for each ray and, after blurring and application of the DQE file, computes the Poisson fluctuations in each energy bin. After this, the images are combined to form an effective path length image. This approach has been described in more detail elsewhere[20].

In Figure 2 we simulate radiographs with bremsstrahlung sources having endpoint energies of $225 \mathrm{keV}$ and $9 \mathrm{MeV}$. In each case, the spectrum was computed in MCNP by colliding electrons of the chosen energy into a tungsten target $1 / 3$ of an electron range thick, generating a 41-energy spectrum model. In both cases, we use a source to object distance of $100 \mathrm{~cm}$

and an object to detector distance of $20 \mathrm{~cm}$. A Gaussian source with $0.15 \mathrm{~cm}$ full width at half maximum was used. We have modeled a Varian dpi $\chi$ flat panel detector computed and reported previously[20]. We have set infinite dose for these examples. The images had a pixel size of $(0.02 \mathrm{~cm})^{2}$ and were $2084^{2}$ in number. Each image took approximately 63 minutes on a Compaq GS 320 machine, which uses a $1 \mathrm{GHz}$ EV68 Alpha chip. The size of the calculation grew to 700 Mbytes during the run because 41 images of size $2084^{2}$ were being kept in memory.

a)

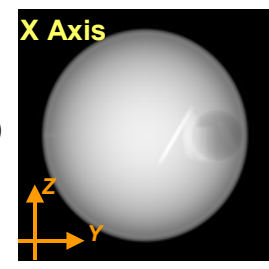

b)

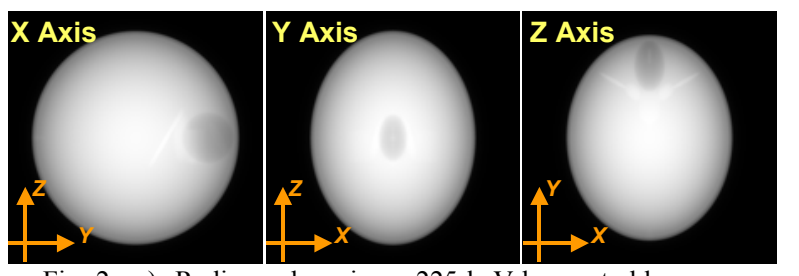

Fig. 2. a) Radiographs using a $225 \mathrm{keV}$ bremsstrahlung source. b) Radiographs using a $9 \mathrm{MeV}$ bremsstrahlung source.

In comparing the images corresponding to each energy, two main trends are apparent. First, the $225 \mathrm{kV}$ case provides better contrast in the images, because this source is not as penetrating. Second, the $9 \mathrm{MV}$ case is significantly blurrier. This blur comes from mainly from electron showers created in the detector by photons of $\mathrm{MeV}$ energies. The minimum transmission in the $225 \mathrm{kV}$ case is 0.005 , while the $9 \mathrm{MV}$ case has minimal transmission of 0.18 .

\section{PROTON RADIOGRAPHY SIMULATION}

The physical processes which protons experience in their passage through matter are very different from what photons experience. The three main processes are attenuation by nuclear interactions, energy loss from scattering off electrons in medium and multiple Coulomb scattering from electrons and nuclei in medium. Nuclear attenuation is the process most analogous to the photon interactions that HADES was originally designed to model. For Hydrogen, we use the proton-proton cross sections[21] and Letaw's fits[15] for all other elements. These cross sections are formed into mass absorption coefficients and integrated along each ray to get a nuclear path length. For energy loss, we use the tables of Ziegler[22], to form energy loss coefficients and compute net energy loss through the objects along each ray. We currently do not integrate energy loss using any slowing down approximation, because HADES computes each object's contribution to each ray in a random order. For multiple 
Coulomb scattering, HADES first computes the radiation length along each ray using Dahl's expression[16]. For each ray, HADES then computes the angular width of the scatter using a standard expression:

$$
\Theta_{0}=\frac{13.6 \mathrm{MeV}}{\beta c p} \sqrt{\int_{\text {ray }} \frac{\rho d l}{X_{0}}},
$$

where $\beta c$ and $p$ are the proton velocity and momentum, respectively, $\mathrm{X}_{0}$ is radiation length and the quantity in the square root is the radiation length along the ray.

a)

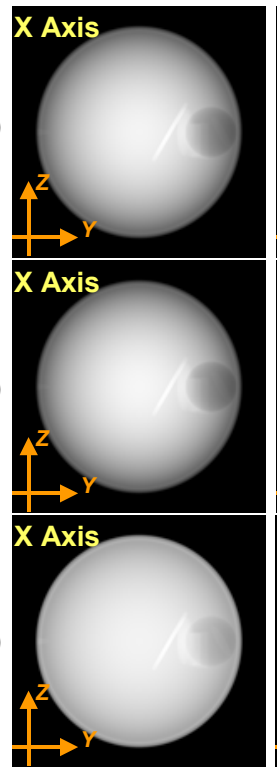

Fig. 3. a) Proton radiography nuclear attenuation image. b) Proton radiography energy loss image. c) Proton radiography angular width image.

HADES computes a nuclear attenuation length image, an energy loss image and a radiation length image that is then used to build an angular width image for the object. Image formation from these quantities depends on how the proton radiography will be done in the experiment. HADES uses the Fermi-Eyges approximation[23] to compute the proton distribution function exiting the object and, if magnetic lenses are used to correct for the effects of multiple Coulomb scattering, then propagates these scattered protons through the magnetic beam line, having included nuclear attenuation and energy loss and spread. In the case of portal imaging using proton therapy beams, a proton telescope would be used just downstream of the object of interest. The proton images would show energy loss, attenuated by nuclear processes and blurred by the scatter of the protons from the object to the detector.

For this paper, we will simply display the nuclear path length, energy loss and angular width of the FORBILD phantom in Figure 3. We have used the same geometry, pixel size, and image size as in the photon examples. We have used $200 \mathrm{MeV}$ protons in the simulation because these will pass through the phantom without extensive deposition, as would be desired for a pretreatment portal image. Each set of images was generated in 2 minutes on an SGI IRIX Origin server running IRIX64 version 6.4 with a clock speed of $195 \mathrm{MHz}$. This calculation was much faster than the photon run because of the size of the photon run.

The minimum transmission due to nuclear attenuation is 0.71 . The maximum energy loss through the object is 84 $\mathrm{MeV}$. The maximum angular width is 56 mradians. All three physical processes distinguish the bone from the tissue better than the photon images because proton interactions are more sensitive to hydrogen content of objects than photons are.

\section{X-RAY PHASE IMAGING SIMULATION}

A new area of research interest is in the use of X-ray micro focus or synchrotron sources, which have sufficient spatial coherence to enable the use of X-ray phase effects in radiography. With high resolution detectors and adequate downstream propagation distance, it is possible to observe the phase structure of mesoscale (less than roughly $0.5 \mathrm{~cm}$ ) objects. For slowly varying structures within such objects, phase effects act like a lens refracting the $\mathrm{X}$-rays and redirecting the energy flow. For more sharply varying objects, diffractive effects can be seen, particularly at interfaces.

In order to model these effects, HADES uses ray tracing to estimate the complex-valued exit wave from the object. HADES uses the atomic scattering factors to compute the phase length $\varphi$ and absorption length $\mu$ through the object. These path lengths are used to form the wave exiting from the object using $\psi(x, y, z=0)=\sqrt{e^{-\mu}} e^{i \varphi}$, where $\mathrm{z}$ is the radiographic axis and $z=0$ is the center of the object. This wave is then propagated to the detector at $\mathrm{z}$ using the Fresnel integral. To compute this integral, HADES uses the convolution form of the integral, which allows the integrals to be performed quickly using FFTs. A more detailed discussion of this work is given in [24].

Currently HADES can only model phase contrast for monochromatic sources with X-Ray energies up to $30 \mathrm{keV}$. Even $30 \mathrm{keV} \mathrm{X}$-rays will not penetrate this phantom. For the purposes of demonstration, we have shrunk the phantom a factor of 100 in all dimensions, so that it is roughly $(0.25 \mathrm{~cm})^{3}$ in size. Although this small object is not relevant as a head phantom, it is the correct size for small biological objects such as frog embryos or tissue samples.

In Figure 4, we use a source energy of $20 \mathrm{keV}$, a source to object distance of $25 \mathrm{~cm}$ and an object to detector distance of 5 $\mathrm{cm}$. We choose $(2.8 \mu \mathrm{m})^{2}$ pixels at the object and a $(1500)^{2}$ image. Each image took 48 minutes to generate on a Dell Precision 420 workstation with a $1 \mathrm{GHz}$ clock speed running Redhat Linux 7.1. The enhancement of interfaces due to diffractive effects is very clear. The minimum transmission of the images is 0.51 . The maximum transmission is 1.4 , greater than one because of constructive interference at the interfaces. This probe has been the most successful at delineating the 
objects in the phantom, but even it has not been able to radiographically distinguish the low contrast features, such as the ventrical, subdural hematoma, or eyes from the brain tissue.

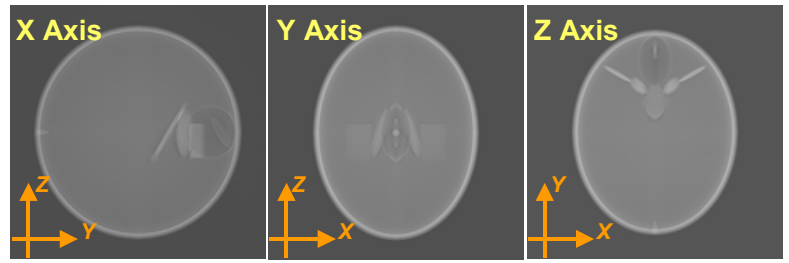

Fig. 4. X-ray phase contrast radiographs at $20 \mathrm{keV}$ for modified head phantom.

\section{CONCLUSIONS AND FUTURE WORK}

In this paper, we have demonstrated HADES' versatility in modeling radiographic probes such as X- and gamma rays, protons and X-ray phase contrast. HADES is also able to model neutron radiography in a treatment analogous to the Xand gamma rays. Development on the code is proceeding in a number of areas.

The phase contrast portion of the code is currently undergoing validation comparisons with data taken at a variety of micro focus and synchrotron systems. This portion of the code needs to be generalized to include polychromatic sources and detector responses. We also need to incorporate the NIST cross section tables so that HADES can model phase effects at higher energy.

Another area of needed work is detector modeling. HADES currently allows detailed detector modeling of X- and gamma rays, but protons and phase contrast use simple number counting approximations. The neutron detector option exists in HADES but has not been rigorously tested. These extensions will require code modification, as well as extensive Monte Carlo studies of the physics involved with these detectors. Building a detector model for HADES requires a large ensemble of Monte Carlo calculations since the detector's blur function and DQE must be mapped over a broad energy range and in fairly small steps in energy.

HADES was written around a transmission radiography paradigm but there are a variety of radiographic problems that do not fit this paradigm, such as backlighting, pinhole imaging, photon scattering, and emission techniques. In order to treat these kinds of problems, we are rewriting HADES in $\mathrm{C}++$ using object-oriented techniques. A fundamental object in this treatment will be the ray, which will have special rules at the start and end, which will allow rays to be created and terminated within the problem and connected to other rays. We will also explore linking HADES and the GEANT Monte Carlo toolkit in order to perform limited scatter calculations during HADES runs.

\section{REFERENCES}

[1] S. Flampouri, P. M. Evans, F. Verhaegen, A. E. Nahum, E. Spezi and M. Partridge, "Optimization of Accelerator Target and Detector for Portal Imaging Using Monte Carlo Simulation and Experiment", Phys. Med. Biol. 47, 3331-3349 (2002).

[2] F. Noo, M. Defrise and H. Kudo, "General Reconstruction Theory for Multislice X-ray Computed Tomography With a Gantry Tilt", IEEE Trans. Med. Im., 23, 1109-1116 (2004).

[3] MCNP4C User's Manual, available through the Radiation Safety Information Computational Center (RSICC). Available online at http://www-rsicc.ornl.gov/ documents/pdf/ccc/ccc7/c700.pdf

[4] T. P. Wilcox, and E. M. Lent, COG User Manual, UC Report Number M-221-1, 1989.

[5] D.E. Cullen, TART-96: A Coupled Neutron-Photon 3-D Combinatorial Geometry Monte Carlo Transport Code, UC Report Number UCRL-ID126455,1996

[6] J.A. Halbleib, and R. P. Kensek, ITS Version 3.0: The Integrated TIGER Series of Coupled Electron/Photon Monte Carlo Transport Codes, Sandia Report SAND91-1634, 1992. Available online at http://www-rsicc.ornl.gov/documents/pdf/ccc/ccc4/c467.pdf

[7] A.F. Bielajew, H. Hirayama W. R. Nelson, and D. W. O. Rogers, History, Overview, and Recent Improvements of EGS4, National Research Council of Canada Report NRC-PIRS-0436, 1994. Available online at http://www-rsicc.ornl.gov/documents/pdf/ccc/ccc3/c331.pdf

[8] D. W. O. Rogers, B. A. Faddegon, G. X. Ding, C.-M. Ma, J. Wei and T. R. Mackie, "BEAM: A Monte Carlo code to simulate radiotherapy treatment units," Med. Phys. 22 (5) 503 - 524, 1995.

[9] S. Agostinelli, J. Allison, K. Amako, J. Apostolakis, H. Araujo, P. Arce, et al., "GEANT4 - a simulation toolkit", NIM A506, 250-303, 2003.

[10] J. Xu, R. M. Wallingford, T. Jensen, and J. N. Gray, "Recent Developments in the X-Ray Radiography Simulation Code: XRSIM", in Review of Progress in Nondestructive Evaluation, 13A, (1994), pp. 557562 .

[11] A. Glière, "SINDBAD: From CAD Model to Synthetic Radiographs", in Review of Progress in Nondestructive Evaluation, 17A, (1998), pp. 387394.

[12] http://www.imp.uni-erlangen.de/forbild/english/results/head/head.html

[13] D. E. Cullen et al., "Tables and Graphs of Photon-Interaction Cross Sections from $10 \mathrm{eV}$ to $100 \mathrm{GeV}$ Derived from the LLNL Evaluated Photon Data Library (EPDL), Parts A and B, UCRL-50400, Vol. 6A and 6B, Rev 4, 1989.

[14] R. J. Howerton et al., "The LLL Evaluated Nuclear Data Library (ENDL): Graphs of Cross Sections from the Library", UCRL050400, Vol. 15, Part B. 1976

[15] J. R. Letaw, R. Silberberg, and C. H. Tsao, Ap. J. Supp., 51, (1983) p.271.

[16] Particle Data Group, "Review of Particle Properties", Physical Review, D66, (2002), p.199.

[17] B. L. Henke, Gullikson, E. M., and Davis, J. C., "X-ray interactions: photoabsorption, scattering, transmission, and reflection at $\mathrm{E}=50-30000$ eV, Z=1-92", Atomic Data and Nuclear Data Tables 54 no.2, 181-342 (July 1993). http://www.cxro.lbl.gov/optical_constants/asf.html

[18] C. T. Chantler, K. Olsen, R. A. Dragoset, A. R. Kishore, S. A. Kotochigova, and D. S. Zucker, (2003), X-Ray Form Factor, Attenuation and Scattering Tables (version 2.0). http://physics.nist.gov/ffast

[19] ICRU Report 10b, "Physical Aspects of Irradiation" (1964).

[20] A. E. Schach von Wittenau, C. M. Logan, M. B. Aufderheide and D. M. Slone, Med. Phys. 29 (11), Nov. 2002, pp. 2559-2570.

[21] http://pdg.lbl.gov/2002/contents_plots.html

[22] J. F. Ziegler, J. P, Biersack, U. Littmark, "The Stopping and Range of Ions in Solids," vol. 1 of series Stopping and Ranges of Ions in Matter, Pergamon Press, New York (1984). See also http://www.srim.org for Ziegler's most recent work.

[23] M. B. Aufderheide, H.-S. Park, E. P. Hartouni, P. D. Barnes, D. M. Wright, R. M. Bionta, J. D. Zumbro and C. L. Morris, "Proton Radiography as a Means of Material Characterization", in Nondestructive Characterization of Materials IX, ed John Green, AIP Conf. Proc. 497, 706-711 (1999). 
[24] M.B Aufderheide, A. Barty and H. E. Martz, Jr., "Simulation Of Phase Effects In Imaging For Mesoscale NDE", in Review of Progress in Quantitative Nondestructive Evaluation, 24A, AIP Conf. Proc. in press.

This work was performed under the auspices of the U.S. Department of Energy by the University of California, Lawrence Livermore National Laboratory under Contract W-7405-Eng-48. 